\title{
UbcH $\mathrm{O}$ overexpression may represent a marker of anaplastic thyroid carcinomas
}

\section{P Pallante', MT Berlingieri', G Troncone'2, M Kruhoffer ${ }^{3}$, TF Orntoft $^{3}$, G Viglietto', A Caleo ${ }^{2}$, I Migliaccio', M Decaussin-Petrucci ${ }^{4}$, M Santoro', L Palombini' ${ }^{2}$ and A Fusco ${ }^{*}, 1,5$}

'Dipartimento di Biologia e Patologia Cellulare e Molecolare clo Istituto di Endocrinologia ed Oncologia Sperimentale del CNR, Facoltà di Medicina e Chirurgia di Napoli, Università degli Studi di Napoli 'Federico Il', via Pansini, 5, 80131 Naples, Italy; '2Dipartimento di Anatomia Patologica e Citopatologia, Facoltà di Medicina e Chirurgia di Napoli, Università di Napoli 'Federico II', via Pansini, 5, 80131 Naples, Italy; 'Department of Clinical Biochemistry, Aarhus University Hospital, Skejby DK 8200 Aarhus N, Denmark; ${ }^{4}$ Service d'Anatomo-Pathologie, Centre Hospitalier Lyon Sud, Pierre Bénite, France; ${ }^{5}$ NOGEC (Naples Oncogenomic Center)-CEINGE, Biotecnologie Avanzate, via Comunale Margherita, 8013 I Naples, Italy

The hybridisation of an Affymetrix HG_U95Av2 oligonucleotide array with RNAs extracted from six human thyroid carcinoma cell lines and a normal human thyroid primary cell culture led us to the identification of the UbcHIO gene that was upregulated by I50fold in all of the carcinoma cell lines in comparison to the primary culture cells of human normal thyroid origin. Immunohistochemical studies performed on paraffin-embedded tissue sections showed abundant UbcHIO levels in thyroid anaplastic carcinoma samples, whereas no detectable UbcHIO expression was observed in normal thyroid tissues, in adenomas and goiters. Papillary and follicular carcinomas were only weakly positive. These results were further confirmed by RT-PCR and Western blot analyses. The block of $\mathrm{UbcHI} O \mathrm{p}$ protein synthesis induced by RNA interference significantly reduced the growth rate of thyroid carcinoma cell lines. Taken together, these results would indicate that $\mathrm{UbcHIO}$ overexpression is involved in thyroid cell proliferation, and may represent a marker of thyroid anaplastic carcinomas.

British Journal of Cancer (2005) 93, 464-47I. doi: I0.1038/sj.bjc.660272I www.bjcancer.com

Published online 2 August 2005

(c) 2005 Cancer Research UK

Keywords: UbcHI0; thyroid; carcinomas; immunohistochemistry

Tumours are the result of the accumulation of different modifications in critical genes involved in the control of cell proliferation. In a large number of carcinomas with worst prognosis, lesions are not diagnosed until the disease is at an advanced stage. Although various therapeutic approaches are followed in clinical practice, most of them are not life saving. Hence, the discovery of ways to diagnose cancer at an early stage and to establish more effective therapies is a critical and urgent issue. To achieve this goal, identification and characterisation of key molecules that participate in carcinogenesis are essential steps. Thyroid neoplasms represent a good model for studying the events involved in epithelial cell multistep carcinogenesis, because they comprise a broad spectrum of lesions with different degrees of malignancy from benign adenomas, which are not invasive and very well differentiated, to the undifferentiated anaplastic thyroid carcinomas, which are very aggressive and always fatal; papillary and follicular carcinomas, the most common forms of thyroid cancer, represent intermediate forms of neoplasia being differentiated and

*Correspondence: Dr A Fusco, Dipartimento di Biologia e Patologia Cellulare e Molecolare, Facoltà di Medicina e Chirurgia di Napoli and, NOGEC (Naples Oncogenomic Center)-CEINGE, Biotecnologie Avanzate, via Pansini 5, 80I3I Napoli, Italy; E-mail: afusco@napoli.com Received 2 March 2005; revised 16 June 2005; accepted 22 June 2005; published online 2 August 2005 having a good prognosis (Hedinger et al, 1989; Wynford-Thomas, 1997).

The involvement of several oncogenes has been demonstrated in papillary thyroid carcinomas. Activation of the RET/PTC oncogene, caused by rearrangements of the RET protooncogene, is detectable in about $30 \%$ of the cases (Grieco et al, 1990; Tallini et al, 1998). Mutations of the B-RAF gene have been demonstrated in almost $40 \%$ of papillary carcinomas (Fukushima et al, 2003). TRK gene rearrangements (Pierotti et al, 1995) and MET gene overexpression are often found in papillary carcinomas (Di Renzo et al, 1992). RAS gene mutations (Suarez et al, 1990) and PAX8PPAR- $\gamma$ rearrangements (Kroll et al, 2000) are frequently detected in tumours of the follicular type. Impairment of the p53 tumour suppressor gene function represents a typical feature of the anaplastic carcinomas (Ito et al, 1992; Dobashi et al, 1993; Donghi et al, 1993; Fagin et al, 1993; Matias-Guiu et al, 1994). Even though critical molecular mechanisms of thyroid carcinogenesis have been clarified, other molecular steps of neoplastic progression need to be investigated.

Therefore, to identify the genes regulated in the process of thyroid carcinogenesis, we analysed a microarray with RNAs extracted from normal human thyroid primary cell culture (NTPC), and six human thyroid carcinoma cell lines of different histotype (one from a follicular carcinoma, three derived from papillary carcinomas and two from anaplastic carcinomas). Our attention was focused on the $\mathrm{UbcH} 10$ gene that was upregulated 
about 150 -fold in all of the cell lines tested by the cDNA microarray. The UbcH10 gene belongs to the $\mathrm{E} 2$ gene family and codes for a protein of $19.6 \mathrm{kDa}$ that is involved in the ubiquitindependent proteolysis. In this pathway, ubiquitin-conjugating enzyme (E2), together with ubiquitin ligase (E3), transfers ubiquitin to specific substrate proteins (Hershko and Ciechanover, 1998; Joazeiro and Weissman, 2000).

The expression level of UbcH10 was extremely low in the normal thyroid primary culture cells, but strong in all the cancerous cell lines. Immunohistochemical and RT-PCR analyses on a large panel of thyroid neoplasms of different histotypes revealed an increased UbcH10 expression in anaplastic thyroid carcinomas, whereas follicular and papillary carcinomas were just weakly positive. The block of UbcH10 protein synthesis by RNA interference inhibited the growth of two thyroid carcinoma cell lines.

\section{MATERIALS AND METHODS}

\section{Cell culture and transfections}

The human thyroid carcinoma cell lines used in this study are: TPC-1 (Tanaka et al, 1987), WRO (Estour et al, 1989), NPA and ARO (Pang et al, 1989), FRO (Fagin et al, 1993), NIM 1 (Zeki et al, 1993), B-CPAP (Fabien et al, 1994), FB-1 (Fiore et al, 1997), FB-2 (Basolo et al, 2002), Kat-4 and Kat-18 (Ain et al, 1997). They were grown in DMEM (Gibco Laboratories, Carlsbad, CA, USA) containing $10 \%$ fetal calf serum (Gibco Laboratories), glutamine (Gibco Laboratories) and ampicillin/streptomycin (Gibco Laboratories) in a $5 \% \mathrm{CO}_{2}$ atmosphere. Normal human thyroid primary culture cells have been established and grown as already described (Curcio et al, 1994). PC Cl 3 cell line derived from Fischer rat thyroid (Fusco et al, 1987) was cultured in modified F12 medium supplemented with $5 \%$ calf serum (Gibco Laboratories) and six growth factors (thyrotropic hormone, hydrocortisone, insulin, transferrin, somatostatin and glycyl-histidyl-lysine; Sigma, St Louis, MO, USA). Thyroid cells were transfected using Lipofectamine reagent (Invitrogen, Carlsbad, CA, USA) according to the manufacturer's instructions. The transfected cells were selected in a medium containing geneticin (G418) (Life Technologies, Italy). For each transfection, several G418-resistant clones and the mass cell population were isolated and expanded for further analysis.

\section{Human thyroid tissue samples}

Neoplastic human thyroid tissues and normal adjacent tissue or the controlateral normal thyroid lobe were obtained from surgical specimens and immediately frozen in liquid nitrogen. Thyroid tumours were collected at the Service d'Anatomo-Pathologie, Centre Hospitalier Lyon Sud, Pierre Bénite, France. The tumour samples were stored frozen until RNA or protein extractions were performed.

\section{RNA isolation}

Total RNA was extracted from tissues and cell cultures using the RNAeasy mini kit (Qiagen, Valencia, CA, USA) according to the manufacturer's instructions. The integrity of the RNA was assessed by denaturing agarose gel electrophoresis.

\section{Reverse transcriptase-PCR analysis}

In total, $5 \mu \mathrm{g}$ of total RNA from each sample, digested with DNAseI (Invitrogen), were reverse transcribed using random hexanucleotides and MuLV reverse transcriptase (Applied Biosystems, Foster City, CA, USA). PCR was carried out on cDNA using the GeneAmp PCR System 9600 (Applied Biosystems). RNA PCR Core Kit (Applied Biosystems) was used to perform RT-PCR reactions.
For the UbcH10 gene, after a first denaturing step $\left(94^{\circ} \mathrm{C}\right.$ for $\left.3 \mathrm{~min}\right)$, PCR amplification was performed for 25 cycles $\left(94^{\circ} \mathrm{C}\right.$ for $30 \mathrm{~s}, 57^{\circ} \mathrm{C}$ for $30 \mathrm{~s}, 72^{\circ} \mathrm{C}$ for $30 \mathrm{~s}$ ). The sequences of forward and reverse primers, amplifying a fragment of $115 \mathrm{bp}$ in the UbcH10 cDNA, were: forward $5^{\prime}$-GCCCGTAAAGGAGCTGAG- $3^{\prime}$ and reverse $5^{\prime}$ GGGAAGGCAGAAATCCCT- $3^{\prime}$. The human $\beta$-actin gene primers, amplifying a $109 \mathrm{bp}$ cDNA fragment, were used as control; amplification was performed for 25 cycles $\left(94^{\circ} \mathrm{C}\right.$ for $30 \mathrm{~s}, 55^{\circ} \mathrm{C}$ for $30 \mathrm{~s}, 72^{\circ} \mathrm{C}$ for $\left.30 \mathrm{~s}\right)$. $\beta$-Actin-forward, $5^{\prime}$-TCGTGCGTGACATTAAG GAG-3 ${ }^{\prime} ; \beta$-actin-reverse, $5^{\prime}$-GTCAGGCAGCTCGTAGCTCT- $3^{\prime}$. To ensure that RNA samples were not contaminated with DNA, negative controls were obtained by performing the PCR on samples that were not reverse-transcribed, but otherwise identically processed. The PCR products were separated on a $2 \%$ agarose gel, stained with ethidium bromide and scanned using a Typhoon 9200 scanner. Quantitative PCR was performed in triplicate using iCycler (Bio-Rad, Hercules, CA, USA) with SYBR ${ }^{\circledR}$ Green PCR Master Mix (Applied Biosystems) as follows: $95^{\circ} \mathrm{C} 10 \mathrm{~min}$ and 40 cycles $\left(95^{\circ} \mathrm{C}\right.$ $15 \mathrm{~s}$ and $\left.60^{\circ} \mathrm{C} 1 \mathrm{~min}\right)$. Fold mRNA overexpression was calculated according to the formula $2^{(\mathrm{Rt}-\mathrm{Et})} / 2^{(\mathrm{Rn}-\mathrm{En})}$ as described previously (El-Rifai et al, 2001), where Rt is the threshold cycle number for the reference gene in the tumour, Et for the experimental gene in the tumor, Rn for the reference gene in the normal sample and En for the experimental gene in the normal sample.

\section{Protein extraction, Western blotting and antibodies}

Cells were washed once in cold PBS and lysed in a lysis buffer containing $50 \mathrm{~mm}$ HEPES, $150 \mathrm{~mm} \mathrm{NaCl}, 1 \mathrm{~mm}$ EDTA, 1 mM EGTA, $10 \%$ glycerol, $1 \%$ Triton-X-100, $1 \mathrm{~mm}$ phenylmethylsulphonyl fluoride, $1 \mu \mathrm{g}$ aprotinin, $0.5 \mathrm{~mm}$ sodium orthovanadate, $20 \mathrm{~mm}$ sodium pyrophosphate. The lysates were clarified by centrifugation at 14000 r.p.m. $\times 10$ min. Protein concentrations were estimated by a Bio-Rad assay (Bio-Rad), and boiled in Laemmli buffer (Tris-HCl pH 6.8, 0.125 M, SDS 4\%, glycerol 20\%, 2-mercaptoethanol $10 \%$, bromophenol blue $0.002 \%$ ) for $5 \mathrm{~min}$ before electrophoresis. Proteins were subjected to SDS - PAGE (15\% polyacrylamide) under reducing condition. After electrophoresis, proteins were transferred to nitrocellulose membranes (Immobilon-P Millipore Corp., Bedford, MA, USA); complete transfer was assessed using prestained protein standards (Bio-Rad). After blocking with TBSBSA (25 mM Tris, pH 7.4, $200 \mathrm{~mm} \mathrm{NaCl,} \mathrm{5 \%} \mathrm{bovine} \mathrm{serum}$ albumin), the membrane was incubated with the primary antibody against UbcH10 (Boston Biochem Inc., Cambridge, MA, USA) for $60 \mathrm{~min}$ (at room temperature). Primary antibody against c-Fos protein (Santa Cruz Biotechnology Inc., Santa Cruz, CA, USA) was used to confirm the specificity of siRNAs against UbcH10 protein. To ascertain that equal amounts of protein were loaded, the Western blots were incubated with antibodies against the $\gamma$-tubulin protein (Sigma). Membranes were then incubated with the horseradish peroxidase-conjugated secondary antibody $(1: 3000)$ for $60 \mathrm{~min}$ (at room temperature) and the reaction was detected with a Western blotting detection system (ECL; Amersham Biosciences, UK).

\section{Immunohistochemistry: tissue samples}

UbcH10 protein cellular distribution was assessed by immunohistochemical analysis and compared to that of the standard cell proliferation marker Ki-67/MIB1. A series of surgical specimens from patients with thyroid diseases comprised of Hashimoto's thyroiditis-HT (six cases), nodular goiter (12 cases), follicular carcinoma (13 cases), papillary carcinoma (33 cases), poorly differentiated carcinoma (five cases) and anaplastic carcinoma (15 cases) was chosen to represent a wide range of thyroid pathology. As control, 10 areas of normal thyroid parenchyma were selected from the lobe controlateral to the tumour in surgical specimens of papillary carcinoma. 
466

\section{Immunostaining: technique, evaluation and statistical} analysis

Xylene dewaxed and alcohol-rehydrated paraffin sections were placed in Coplin jars filled with a $0.01 \mathrm{~m}$ tri-sodium citrate solution, and heated for $3 \mathrm{~min}$ in a conventional pressure cooker (Troncone et al, 2003). After heating, slides were thoroughly rinsed in cool running water for $5 \mathrm{~min}$. They were then washed in Tris-Buffered Saline (TBS) pH 7.4 before incubating overnight with the specific antibody, diluted as follows: rabbit polyclonal $\alpha$-UbcH10 (Boston Biochem) 1:1000; $\alpha$-MIB-1 (Novocastra, Newcastle upon Tyne, UK) 1:50. After incubation with the primary antibody, tissue sections were stained with biotinylated anti-rabbit or anti-mouse immunoglobulins, followed by peroxidase-labelled streptavidine (Dako, Carpinteria, CA, USA); the signal was developed by using diaminobenzidine $(\mathrm{DAB})$ chromogen as substrate. Incubations both omitting the specific antibody, and including unrelated antibodies, were used as negative controls.

Individual cells were scored for expression of UbcH10 and Ki-67 in similar areas of adjacent sections by quantitative analysis performed with a computerised analyser system (Ibas 2000, Kontron, Zeiss), as already described (Troncone et al, 2003). In each case, the distribution of these proteins was evaluated in at least 500 epithelial follicular cells and expressed as a percentage of the total cell population. The statistical analysis was performed using SPSS Ver. 9.0.1 for Windows'. Data are expressed as median value and range. The nonparametric Mann-Whitney $U$-test was used to compare differences in labelling indexes for UbcH10 and Ki-67 in thyroid carcinomas. The Spearman rank order correlation was used to verify the association between UbcH10 and Ki-67. A $P$-value less than 0.05 was considered statistically significant.

\section{RNA interference}

For small interfering RNA (siRNA) experiments, the following double-strand RNA oligo specific for UbcH10 coding region was used: $5^{\prime}$-AACCTGCAAGAAACCTACTCA- $3^{\prime}$ as previously described (Wagner et al, 2004). As negative control, we used a corresponding scrambled sequence as follows: $5^{\prime}$-AACTAACAC TAGCTCAAGACC- $3^{\prime}$. All of the siRNA duplexes were purchased from Qiagen and were transfected using Oligofectamine (Invitrogen) according to the manufacturer's recommendations. Small interfering RNAs were used at a final concentration of $100 \mathrm{nM}$ and $12 \times 10^{5}$ cells well $^{-1}$ were plated in a six-well format plates. Proteins were extracted $48 \mathrm{~h}$ after siRNA treatment and the levels of the UbcH10 protein were evaluated by Western blot.

\section{Assay of the transformed state}

Tumorigenicity of the cell lines was tested by injecting $2 \times 10^{6}$ cells subcutaneously into athymic mice. Soft agar colony assay was performed as previously described (Macpherson and Montagnier, 1964).

\section{RESULTS}

Expression of UbcH10 gene in normal human thyroid cells and thyroid carcinoma cell lines

To search for candidate genes involved in the neoplastic transformation of thyroid gland, RNAs extracted from normal human thyroid primary cells and six human thyroid carcinoma cell lines of different origin (WRO cell line from a follicular carcinoma, TPC-1 and FB-2 cell lines, both deriving from papillary thyroid cancers, NPA cell line, which derives from a poorly differentiated papillary carcinoma, ARO and FRO cell lines originating from anaplastic carcinomas) were hybridised to U95Av2 Affymetrix oligonucleotide arrays (data not shown). We
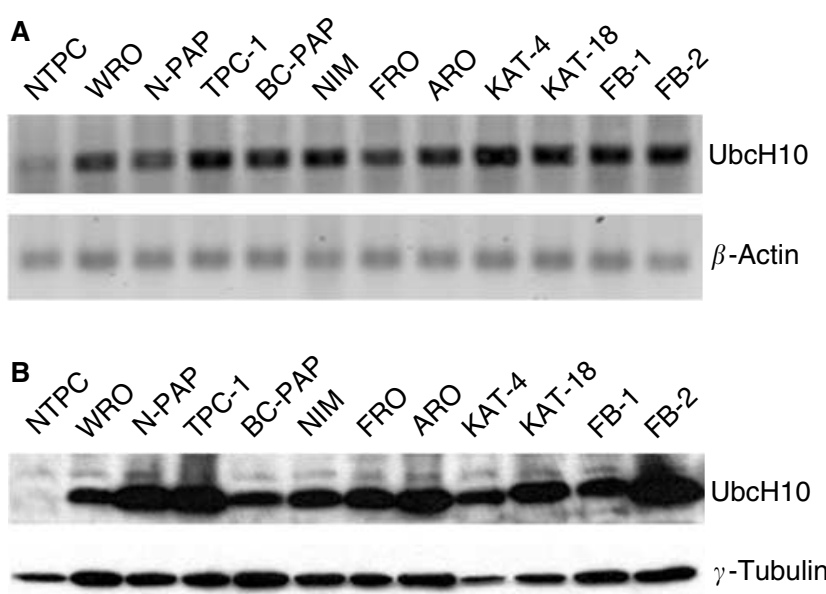

Figure I UbcHIO expression in human thyroid carcinoma cell lines. (A) UbcHIO gene expression analysis by RT-PCR in human thyroid carcinoma cell lines vs the normal human thyroid primary culture cells (NTPC). $\beta$ Actin gene expression was evaluated as control to normalise the amount of the used RNAs. (B) UbcHIO protein expression analysis by Western Blot in human thyroid carcinoma cell lines. Blot against $\gamma$-tubulin has been performed as control for equal protein loading.

concentrated our attention on the UbcH10 gene that was upregulated about 150 -fold in all of the cell lines tested by the cDNA microarray. This result was confirmed by RT-PCR in a larger panel of thyroid carcinoma cell lines using as control normal thyroid primary culture (Figure 1A). Western blot analysis of UbcH10 expression, shown in Figure 1B, confirmed the RT-PCR data. In fact, the UbcH10 protein was abundantly expressed in all of the carcinoma cell lines, whereas it was barely detectable in normal thyroid cells.

\section{Analysis of UbcH10 expression in normal and neoplastic thyroid tissues by immunohistochemistry, Western blot and RT - PCR}

In order to evaluate whether the overexpression of $\mathrm{UbcH} 10$ is a feature of the thyroid tumours and not only of cultured thyroid carcinoma cell lines, we performed immunohistochemical analysis using a commercial antibody against $\mathrm{UbcH} 10$ protein. This methodology allows a rapid and sensitive screening of thyroid pathological tissues and is amenable to regular use as a routine diagnostic test. To find the best experimental conditions, ARO cell line and tumours, induced by injecting the ARO cell line into athymic mice, were used as positive controls (Cerutti et al, 1996). No staining was observed with normal human thyroid primary cell culture, whereas a positive staining was obtained with ARO cell line and ARO-induced tumours (data not shown). We found that normal thyroid, nodular goiter and Hashimoto's thyroiditis (HT) were almost always completely negative for UbcH10 expression. Only occasionally, single UbcH10-labelled thyroid epithelial cells showing mitotic figures could be observed by meticulous scrutiny (Figure 2A). In HT, there was a sharp contrast between the negative epithelial oxyphilic cells and the positive lymphoid germinal centers (Figure 2B). While a weak staining is detectable in follicular adenomas (Figure 2C), higher levels of UbcH10 were recorded in papillary (median value $2.2 \%$ of positive cells; range $0.9-4.1 \%$ ), follicular (median value $2.8 \%$ of positive cells; range 1 $6.1 \%$ ) and poorly differentiated (median value $10.4 \%$ of positive cells; range $8-14.9 \%$ ) carcinomas, signal being always easily detectable in the nuclei of scattered neoplastic cells (Figure 2D, E and F). UbcH10 staining pattern was somewhat different in 

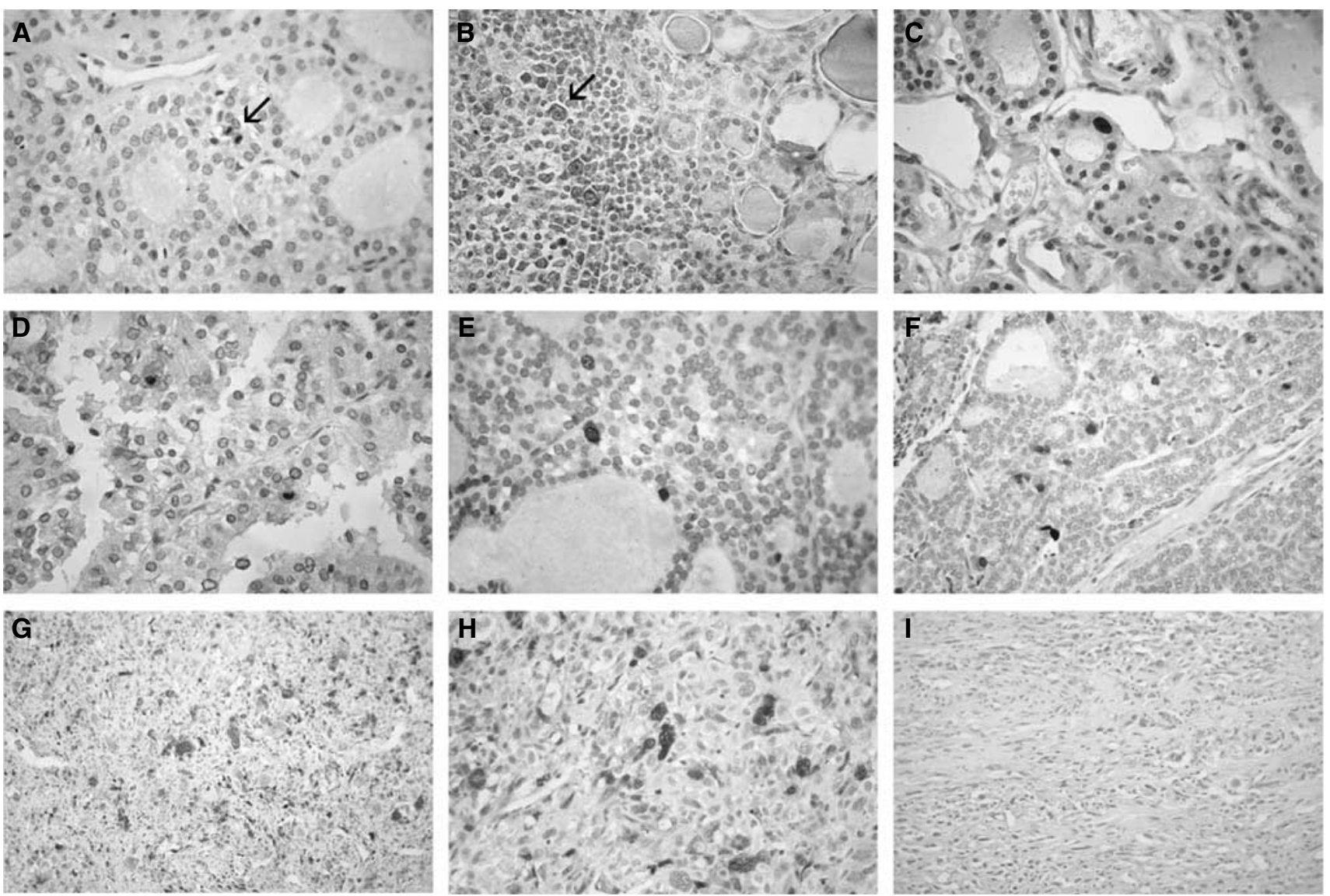

Figure 2 UbchIO staining pattern in normal, inflammatory and neoplastic thyroid tissues. Follicular epithelial cells of normal thyroid (A) and oxyphilic cells of Hashimoto's thyroiditis (HT) (B) do not stain for UbcHIO, with occasional mitotic figures (A, arrow) and lymphoid centroblasts of HT (B, arrow) providing the appropriate internal positive control. In neoplastic thyroid, $\mathrm{UbcHI} O \mathrm{staining}$ pattern is strongly related to tumour grade, being weak in follicular adenoma $(\mathbf{C})$, slightly more evident in well-differentiated papillary $(\mathbf{D})$ and follicular $(\mathbf{E})$ carcinomas, whereas stronger in poorly differentiated $(\mathbf{F})$ and in anaplastic $(\mathbf{G})$ carcinomas. In the latter, most of neoplastic cells show a very intense labelling, with intense nuclear staining $(\mathbf{H})$, whereas signal disappeared by antigen incubation (I).

anaplastic carcinomas, the percentage (median value $45.8 \%$ of positive cells; range $38.8-56.2 \%$ ) of stained cells being large and the intensity of the neoplastic cells being strong (Figure $2 \mathrm{G}$ and $\mathrm{H}$ ).

No staining was observed when the same anaplastic carcinoma samples were stained with antibodies preincubated with $\mathrm{UbcH} 10$ recombinant protein (Figure 2I) or in the absence of the primary antibodies (data not shown). Therefore, as a general rule, UbcH10 expression is negligible in non-neoplastic thyroid, noticeable in well-differentiated carcinomas and conspicuous in less-differentiated tumours (Figure 3A).

To determine the relationship between $\mathrm{UbcH} 10$ expression and tissue proliferation, we correlated its expression in carcinomas with the proliferation rate of thyrocytes, as measured by $\mathrm{Ki}-67$ staining; this latter showed the same tissue distribution of $\mathrm{UbcH} 10$, which was evident when adjacent (mirror) sections were stained. By using the Spearman rank order correlation, we determined that the association between $\mathrm{UbcH} 10$ and $\mathrm{Ki}-67$ expression in thyroid cancer was statistically significant. In fact, the value of the Spearman $R$ was $0.4(P<0.001)$ (Figure 3B).

Western blot analysis, performed on 30 surgically removed thyroid tumours, confirmed the immunohistochemical data. A representative Western blot is shown in Figure 4A. A strong band of $19.6 \mathrm{kDa}$ corresponding to the $\mathrm{UbcH} 10$ protein was detected in anaplastic thyroid carcinomas and a weak one in poorly differentiated carcinomas, but not in papillary carcinomas and normal thyroids. These data strongly indicate that the expression of UbcH10 is more abundant in thyroid carcinomas characterised by a highly malignant and aggressive phenotype. Equal amounts of total proteins were used for each sample as demonstrated by the same gel analysed with an antibody against $\gamma$-tubulin.

UbcH10 expression was also evaluated by RT - PCR analysis on a panel of matched tumour/normal tissues. This analysis confirmed the protein data. In fact, as shown in Figure 4B, an amplified band of $115 \mathrm{bp}$ was clearly detected in the anaplastic and poorly differentiated carcinoma samples, but not in the papillary ones and in all the corresponding normal thyroid tissues. Finally, quantitative RT-PCR analysis confirmed a great increase of UbcH10 expression in thyroid anaplastic samples, whereas a light increase was observed in papillary carcinoma samples (Figure 4C).

\section{UbcH10 expression in experimental models of thyroid carcinogenesis}

Thyroid neoplasias developing in transgenic animal lines expressing TRK (Tg-TRK) (Russell et al, 2000), RET/PTC3 (Tg-RET/ PTC3) (Powell et al, 1998) and large T SV40 (Tg-SV40) (Ledent et al, 1991) oncogenes under the transcriptional control of the thyroglobulin promoter have been analysed for UbcH10 expression by Western blot analysis. Transgenic mice carrying TRK and RET/PTC3 oncogenes develop thyroid papillary carcinomas (Powell et al, 1998; Russell et al, 2000); thyroid anaplastic carcinomas were, conversely, obtained in the Tg-SV40 mice 


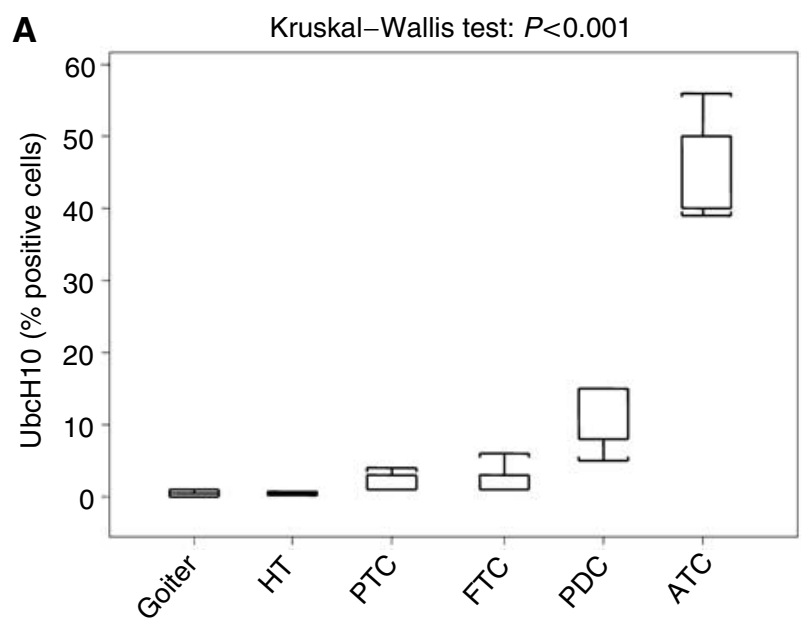

B Spearman's $\rho: 0.4 ; P<0.001$

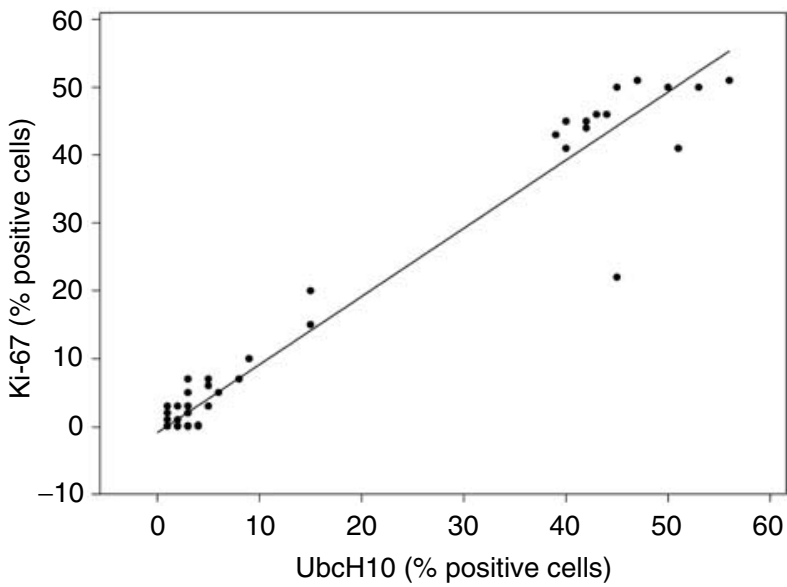

Figure 3 Statistical analysis of the immunohistochemical data. (A) Protein expression of $\mathrm{UbcHIO}$ (\% positive cells) progressively increases in the several diagnostic categories from thyroid goiter to the thyroid anaplastic carcinomas. The analysis has been carried out using the KruskalWallis test. HT, Hashimoto's thyroiditis; PTC, papillary thyroid carcinoma; FTC, follicular thyroid carcinoma; PDC, poorly differentiated thyroid carcinoma; ATC, anaplastic thyroid carcinoma. (B) Protein expression of $\mathrm{UbcHIO}$ (\% positive cells) is correlated to that of Ki-67 (\% positive cells) in the several diagnostic categories. The analysis has been carried out calculating the Spearman rank correlation coefficient.

(Ledent et al, 1991). As shown in Figure 5, elevated UbcH10 protein levels were observed in the thyroid anaplastic carcinomas derived from large TSV40 transgenic mice. Conversely, UbcH10 protein was absent in normal mouse thyroid tissue and in the papillary carcinomas originating from TRK and RET/PTC3 mice.

Therefore, the analysis of the experimental models of thyroid carcinogenesis seems to confirm that the UbcH10 overexpression is essentially restricted to the undifferentiated histotype.

\section{Suppression of the UbcH10 synthesis inhibits thyroid carcinoma cell growth}

We asked whether UbcH10 overexpression had a role in the process of thyroid carcinogenesis by evaluating the growth rate of two thyroid carcinoma cell lines, in which $\mathrm{UbcH} 10$ protein was suppressed by RNA interference. The NPA and TPC-1 cell lines were treated with siRNA duplexes targeting to the UbcH10 mRNA. After transfection, we observed an efficient knockdown of the UbcH10 protein levels at $48 \mathrm{~h}$ after treatment (Figure 6A). The
A

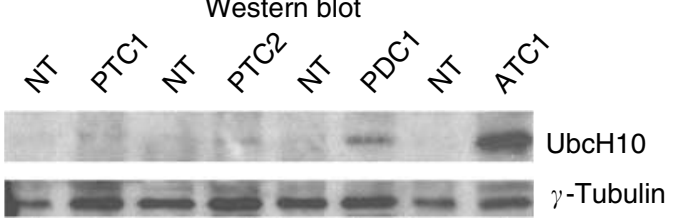

B RT-PCR

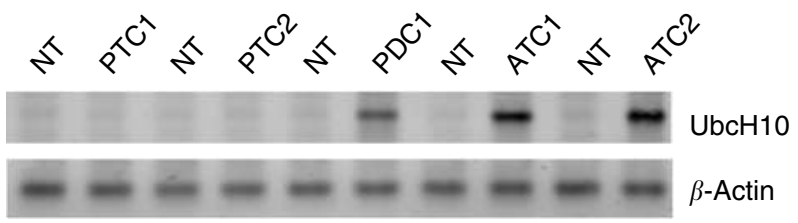

C

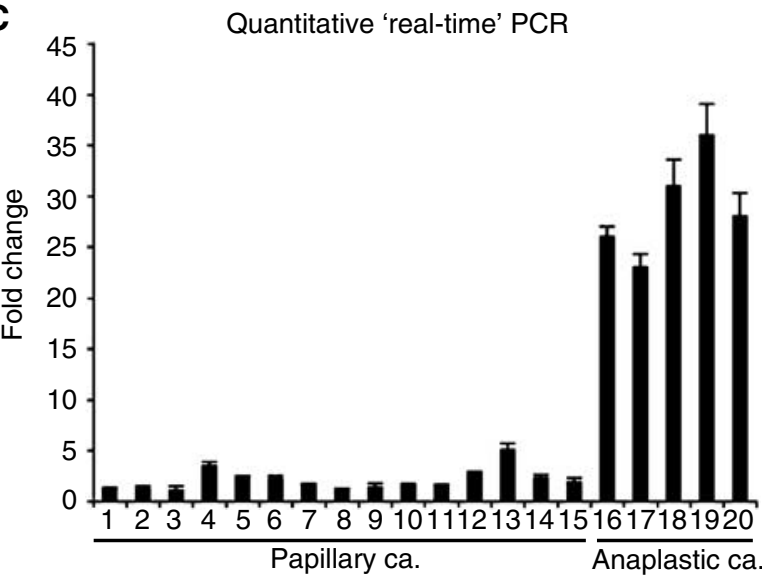

Figure $4 \mathrm{UbcHIO}$ expression in human thyroid fresh tumour samples. (A) Western blot analysis of $\mathrm{UbcHIO}$ protein expression in a panel of thyroid neoplasias. The level of $\gamma$-tubulin has been used as loading control. NT, normal thyroid tissue; PTCl and PTC2, papillary thyroid carcinomas from two different patients; PDCI, poorly differentiated carcinoma; ATCI, anaplastic thyroid carcinoma. (B) RT-PCR analysis of $\mathrm{UbcH} O \mathrm{O}$ expression in human thyroid tumour samples vs their normal thyroid counterparts. $\beta$ Actin expression shows the same amount of RNAs used. NT, normal thyroid tissue; PTCI and PTC2, papillary thyroid carcinomas from two different patients; $\mathrm{PDCl}$, poorly differentiated carcinoma; ATCI and ATC2, anaplastic thyroid carcinomas from two different patients. (C) Quantitative RT-PCR analysis was performed on human thyroid tumour samples of different histotype. The Fold Change values indicate the relative change in the expression levels between tumour samples and normal samples, assuming that the value of each normal sample is equal to I.

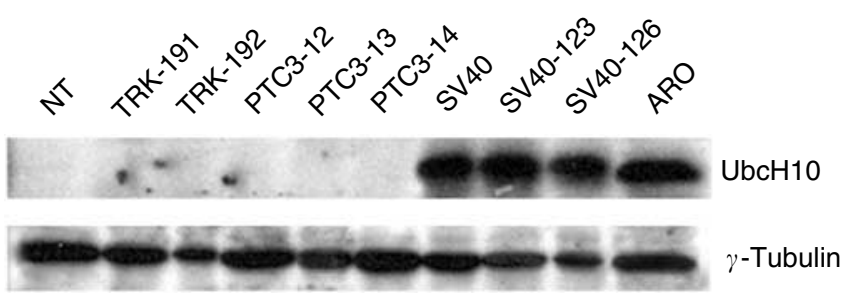

Figure $5 \mathrm{UbcH} / \mathrm{O}$ expression in experimental mouse thyroid tumours Western blot analysis of experimental thyroid carcinomas developed in transgenic mice expressing TRK, RET-PTC-3 and large T SV40 oncogenes. ARO cell line was used as positive control. $\gamma$-Tubulin shows the same amount of protein level.

analysis of cell growth of these cell lines in the presence or absence of the UbcH10 siRNA duplexes revealed that the block of the UbcH10 protein synthesis significantly inhibits thyroid carcinoma 
A
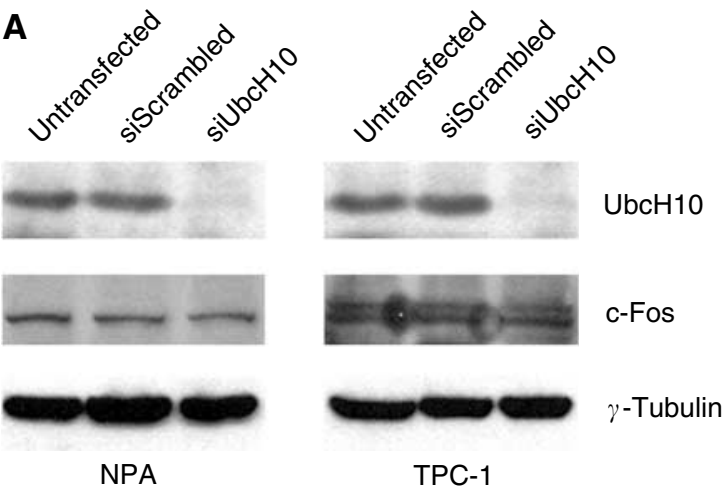

NPA

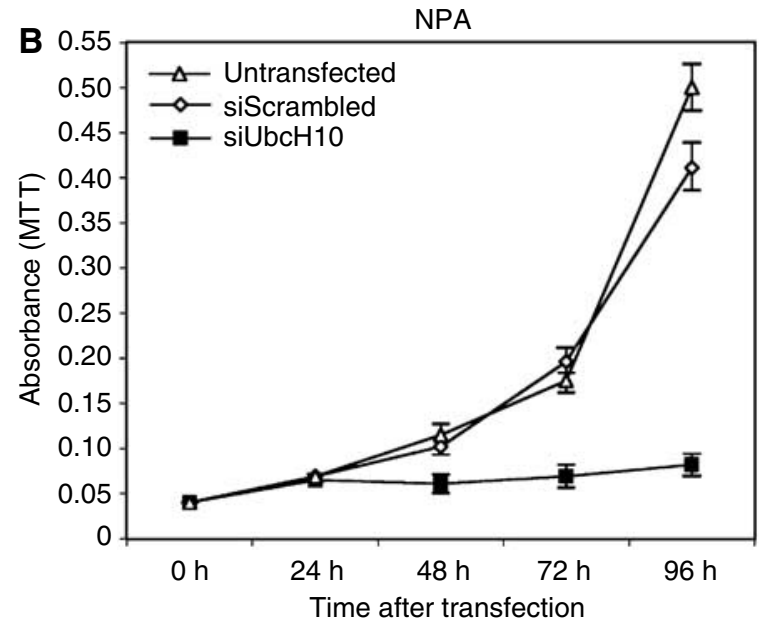

TPC-1

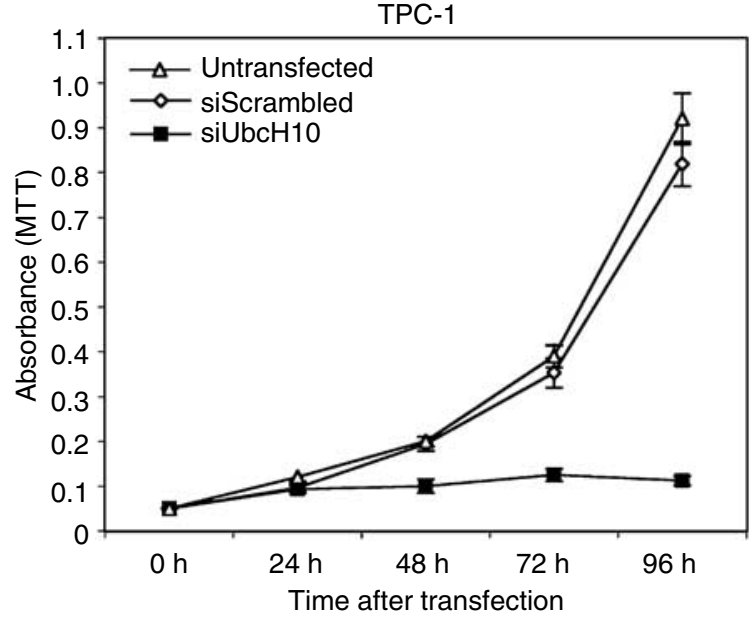

Figure $\mathbf{6}$ The block of $\mathrm{UbcHIO}$ protein synthesis by RNA interference inhibits the proliferation of thyroid carcinoma cells. (A) Inhibition of UbcHIO protein expression by RNAi in NPA and TPC-I cell lines evaluated by Western blot analysis. At $48 \mathrm{~h}$ after siRNA transfection, total cell lysates were prepared and normalised for protein concentration. The expression of $\gamma$-tubulin was used to control equal protein loading $(30 \mu \mathrm{g})$. In this figure, we also show the expression of a fast turning-over gene like cFos to confirm the specific effect of siRNAs against UbcHIO. (B) Growth curves of NPA and TPC-I cell lines after siUbcHIO treatment. NPA and TPC-I cells were transfected with siUbcHIO duplexes $($ siUbcHIO) and the relative number of viable cells was determined by MTT assay. Cells transfected with a scrambled duplex (siScrambled) and untransfected cells (Untransfected) were used as negative controls. Absorbance was read at $570 \mathrm{~nm}$ and the data are the mean of triplicates.
Table I Analysis of the neoplastic phenotype of the UbcHIOtransfected rat thyroid cell lines

\begin{tabular}{lccc}
\hline Cell line & $\begin{array}{c}\text { Doubling time } \\
\text { (h) }\end{array}$ & $\begin{array}{c}\text { Colony- } \\
\text { forming } \\
\text { efficiency (\%) }\end{array}$ & $\begin{array}{c}\text { Tumour }^{\mathbf{b}} \\
\text { incidence }^{-}\end{array}$ \\
\hline PC CL 3 & 24 & 0 & $0 / 4$ \\
PC UbCHIO CLI & 24 & 0 & $0 / 4$ \\
PC UbCHIO CL2 & 23 & 0 & $0 / 4$ \\
PC MPSV & 18 & 70 & $4 / 4$ \\
\hline
\end{tabular}

${ }^{a}$ Colony-forming efficiency was calculated by the formula (number of colonies formed/number of plated cells $) \times 100$. ' $T$ Tumorigenicity was assayed by injecting $2 \times 10^{6}$ cells into athymic mice ( $4-6$ weeks old).

cell growth. In fact, as shown in Figure 6B, a significant reduction in cell growth rate was observed in NPA and TPC-1 cell lines treated with UbcH10 siRNA in comparison to the untreated cells or those treated with the control scrambled siRNA.

These results indicate a role of $\mathrm{UbcH} 10$ in neoplastic thyroid cell proliferation.

\section{UbcH10 overexpression is not sufficient to transform rat thyroid cells}

To further characterise the role of UbcH10 in thyroid carcinogenesis, we transfected normal rat thyroid cells with an expression vector carrying the $\mathrm{UbcH} 10$ gene under the transcriptional control of the cytomegalovirus promoter. The selected clones were shown to express high UbcH10 protein levels (data not shown). We evaluated the growth rate of the UbcH10-transfected PC $\mathrm{Cl} 3$ cells and the same cells transfected with a backbone vector: no differences were observed. Equally, the neoplastic phenotype of the UbcH10-transfected PC Cl 3 cells was evaluated by a soft agar colony assay and by injection into athymic mice. As reported in Table 1, the PC Cl 3 cells transfected with the UbcH10 expression vector were not able to give rise to colonies in soft agar and induce tumours in athymic mice. As a positive control, we used the $\mathrm{PC} \mathrm{Cl}$ 3 cells transformed with the Myeloproliferative sarcoma virus (PC MPSV): these cells have a very highly malignant phenotype (Fusco et al, 1987).

These results indicate that $\mathrm{UbcH} 10$ overexpression is not able to transform rat thyroid cells in vitro.

\section{DISCUSSION}

Thyroid neoplasms represent an excellent model for studying the process of cell transformation since they include a broad spectrum of histotypes showing different degree of malignancy (Hedinger et al, 1989; Wynford-Thomas, 1997). From the Affymetrix microarray analysis, we found the $\mathrm{UbcH} 10$ gene that appeared greatly increased in all of the thyroid carcinoma cell lines. UbcH10 was previously identified as a human homologue of the cyclinselective E2 (E2-c), which is required for the destruction of mitotic cyclins by the ubiquitination pathway. The $\mathrm{UbcH} 10$ gene belongs to the E2 gene family and codes for a protein of $19.6 \mathrm{kDa}$ that is involved in the ubiquitin-dependent proteolysis. In this system, three distinct enzymes cooperate to process target proteins for degradation. More precisely, the ubiquitin-conjugating enzyme (E2) transfers activated ubiquitin by ubiquitin-activating enzyme (E1) to a lysine residue of the target proteins in cooperation with the ubiquitin-ligase (E3). Polyubiquitinated proteins are then recognised by the $26 \mathrm{~S}$ proteasome and rapidly degraded (Hershko and Ciechanover, 1998; Joazeiro and Weissman, 2000).

In our study, we have evaluated the expression of $\mathrm{UbcH} 10$ in human thyroid neoplasias and in mouse experimental tumours. No 
significant UbcH10 expression was observed in normal thyroids, goiters and adenomas, whereas a great induction of UbcH10 expression was observed in anaplastic human thyroid carcinomas and in experimental undifferentiated thyroid tumours. Just a weak expression of UbcH10 was observed in follicular and papillary human thyroid carcinomas. Therefore, these data strongly indicate that $\mathrm{UbcH} 10$ overexpression could be associated with the thyroid tumour progression since there is a good correlation with the late stage of thyroid neoplastic transformation. The low UbcH10 levels detected in the differentiated thyroid malignancies would appear in contrast with the data showing an abundant $\mathrm{UbcH} 10$ expression in the cell lines deriving from differentiated carcinomas. We retain this discrepancy only apparent since thyroid carcinoma cell lines, even deriving from differentiated tumours, cannot be completely compared to surgically removed tumours. In fact, these cell lines harbour p53 mutations that are rare in thyroid differentiated neoplasias (Tanaka et al, 1987; Estour et al, 1989; Pang et al, 1989; Ito et al, 1992; Dobashi et al, 1993; Donghi et al, 1993; Fagin et al, 1993; Zeki et al, 1993; Fabien et al, 1994; Matias-Guiu et al, 1994; Ain et al, 1997; Fiore et al, 1997; Basolo et al, 2002) and they have a high proliferation rate. However, this consideration does not exclude the validity of the use of the thyroid carcinoma cell lines as experimental model to draw new information that, however, need to be subsequently validated on fresh tumours.

Our results also indicate a correlation between $\mathrm{UbcH} 10$ overexpression and the proliferation status since there is a good association with the proliferation marker Ki-67/MIB1.

An aim of our work was to evaluate the possible causal role of $\mathrm{UbcH} 10$ overexpression in thyroid carcinogenesis. Indeed, the block of protein synthesis significantly inhibited the growth of several thyroid carcinoma cell lines, suggesting an important role of UbcH10 in thyroid cell proliferation, and then in the progression step of thyroid carcinogenesis.

Therefore, even though the mechanisms by which UbcH10 overproduction contributes to the neoplastic phenotype remains unclear, we can assume that it leads to a deregulation of cell growth. These results are quite consistent with previous published data showing that $\mathrm{UbcH} 10$ was expressed at high levels in primary tumours derived from the lung, stomach, uterus, and bladder as compared with their corresponding normal tissues, suggesting that $\mathrm{UbcH} 10$ is involved in tumorigenesis or cancer progression (Okamoto et al, 2003; Wagner et al, 2004). It has also been shown that $\mathrm{UbcH} 10$ is upregulated in NIH3T3 cell line transformed by
EWS/FLI1, but not in untransformed NIH3T3 cell clone expressing EWS/FLI1 (Arvand et al, 1998). Moreover, it has been shown that UbcH10 is required for override metaphase, likely degrading growth suppressor, and for destruction of mitotic cyclins, indicating a role of $\mathrm{UbcH} 10$ in cell cycle progression (Arvand et al, 1998).

However, overexpression of UbcH10 in normal rat thyroid cells did not affect cell growth neither induced a malignant phenotype, indicating that UbcH10 overexpression is not sufficient for malignant thyroid cell transformation. This result might appear in contrast with those showing that NIH3T3 stable transfectants overexpressing $\mathrm{UbcH} 10$ exhibited a malignant phenotype as compared with parental NIH3T3 cells (Okamoto et al, 2003). This discrepancy is, in our opinion, just apparent since we have to consider that NIH $3 \mathrm{~T} 3$ cells are preneoplastic cells, whereas $\mathrm{PC} \mathrm{Cl} 3$ cells are much more resistant to express the neoplastic phenotype, since even the expression of several oncogenes (v-ras-Ki, v-ras-Ha, etc.) are not able to lead these cells to the fully malignant phenotype that is achieved only when there is a synergy of two different oncogenes (Fusco et al, 1987). In conclusion, our data propose the $\mathrm{UbcH} 10$ overexpression as a feature of the anaplastic carcinoma histotype. The block of $\mathrm{UbcH} 10$ expression significantly reduced the growth of thyroid carcinoma cell lines indicating an involvement of $\mathrm{UbcH} 10$ in the increased proliferation of these carcinoma cell lines. Therefore, these results open the perspective of a therapy of the anaplastic thyroid carcinoma, one of the most aggressive tumours in mankind, based on the suppression of the UbcH10 synthesis and/or function.

\section{ACKNOWLEDGEMENTS}

This work was supported by grants from the Associazione Italiana Ricerca sul Cancro (AIRC), Progetto Strategico Oncologia Consiglio Nazionale delle Ricerche, the Ministero dell'Università e della Ricerca Scientifica e Tecnologica (MIUR), and 'Piani di Potenziamento della Rete Scientifica e Tecnologica' CLUSTER C04, the Programma Italia-USA sulla Terapia dei Tumori coordinated by Professor Cesare Peschle, and 'Ministero della Salute'. This work was supported from NOGEC-Naples Oncogenomic Center. We thank the Associazione Partenopea per le Ricerche Oncologiche (APRO) for its support. We are grateful to Jean Ann Gilder (Scientific Communication) for editing the text.

\section{REFERENCES}

Ain KB, Taylor KD, Tofiq S, Venkataraman G (1997) Somatostatin receptor subtype expression in human thyroid and thyroid carcinoma cell lines. J Clin Endocrinol Metab 82: $1857-1862$

Arvand A, Bastians H, Welford SM, Thompson AD, Ruderman JV, Denny CT (1998) EWS/FLI1 up regulates mE2-C, a cyclin-selective ubiquitin conjugating enzyme involved in cyclin B destruction. Oncogene 17: 2039-2045

Basolo F, Giannini R, Toniolo A, Casalone R, Nikiforova M, Pacini F, Elisei R, Miccoli P, Berti P, Faviana P, Fiore L, Monaco C, Pierantoni GM, Fedele M, Nikiforov YE, Santoro M, Fusco A (2002) Establishment of a non-tumorigenic papillary thyroid cell line (FB-2) carrying the RET/ PTC1 rearrangement. Int J Cancer 97: 608-614

Cerutti J, Trapasso F, Battaglia C, Zhang L, Martelli ML, Visconti R, Berlingieri MT, Fagin JA, Santoro M, Fusco A (1996) Block of c-myc expression by antisense oligonucleotides inhibits proliferation of human thyroid carcinoma cell lines. Clin Cancer Res 2: 119-126

Curcio F, Ambesi-Impiombato FS, Perrella G, Coon HG (1994) Long-term culture and functional characterization of follicular cells from adult normal human thyroids. Proc Natl Acad Sci USA 91: 9004-9008

Di Renzo MF, Olivero M, Ferro S, Prat M, Bongarzone I, Pilotti S, Belfiore A, Costantino A, Vigneri R, Pierotti MA (1992) Overexpression of the c-MET/HGF receptor gene in human thyroid carcinomas. Oncogene 7: $2549-2553$
Dobashi Y, Sakamoto A, Sugimura H, Mernyei M, Mori M, Oyama T, Machinami R (1993) Overexpression of p53 as a possible prognostic factor in human thyroid carcinoma. Am J Surg Pathol 17: $375-381$

Donghi R, Longoni A, Pilotti S, Michieli P, Della Porta G, Pierotti MA (1993) Gene p53 mutations are restricted to poorly differentiated and undifferentiated carcinomas of the thyroid gland. J Clin Invest 91: $1753-1760$

El-Rifai W, Frierson Jr HF, Moskaluk CA, Harper JC, Petroni GR, Bissonette EA, Jones DR, Knuutila S, Powell SM (2001) Genetic differences between adenocarcinomas arising in Barrett's esophagus and gastric mucosa. Gastroenterology 121: 592-598

Estour B, Van Herle AJ, Juillard GJ, Totanes TL, Sparkes RS, Giuliano AE Klandorf H (1989) Characterization of a human follicular thyroid carcinoma cell line (UCLA RO 82 W-1). Virchows Arch B Cell Pathol Incl Mol Pathol 57: 167-174

Fabien N, Fusco A, Santoro M, Barbier Y, Dubois PM, Paulin C (1994) Description of a human papillary thyroid carcinoma cell line. Morphologic study and expression of tumoral markers. Cancer 73: 2206-2212

Fagin JA, Matsuo K, Karmakar A, Chen DL, Tang SH, Koeffler HP (1993) High prevalence of mutations of p53 gene in poorly differentiated human thyroid carcinomas. J Clin Invest 91: 179-184 
Fiore L, Pollina LE, Fontanini G, Casalone R, Berlingieri MT, Giannini R, Pacini F, Miccoli P, Toniolo A, Fusco A, Basolo F (1997) Cytokine production by a new undifferentiated human thyroid carcinoma cell line, FB-1. J Clin Endocrinol Metab 82: 4094-4100

Fukushima T, Suzuki S, Mashiko M, Ohtake T, Endo Y, Takebayashi Y, Sekikawa K, Hagiwara K, Takenoshita S (2003) BRAF mutations in papillary carcinomas of the thyroid. Oncogene 22: 6455-6457

Fusco A, Berlingieri MT, Di Fiore PP, Portella G, Grieco M, Vecchio G (1987) One- and two-step transformation of rat thyroid epithelial cells by retroviral oncogenes. Mol Cell Biol 7: 3365-3370

Grieco M, Santoro M, Berlingieri MT, Melillo RM, Donghi R, Bongarzone I, Pierotti MA, Della Porta G, Fusco A, Vecchio G (1990) PTC is a novel rearranged form of the RET proto-oncogene and is frequently detected in vivo in human thyroid papillary carcinomas. Cell 60: 557-563

Hedinger C, Williams D, Sobin LH (1989) The WHO histological classification of thyroid tumours: a commentary on the second edition. Cancer 63: 908 -911

Hershko A, Ciechanover A (1998) The ubiquitin system. Annu Rev Biochem 67: $425-479$

Ito T, Seyama T, Mizuno T, Tsuyama N, Hayashi T, Hayashi Y, Dohi K, Nakamura N, Akiyama M (1992) Unique association of p53 mutations with undifferentiated but not differentiated carcinomas of the thyroid gland. Cancer Res 52: 1369-1371

Joazeiro CA, Weissman AM (2000) RING finger proteins: mediators of ubiquitin ligase activity. Cell 102: 549-552

Kroll TG, Sarraf P, Pecciarini L, Chen CJ, Mueller E, Spiegelman BM, Fletcher JA (2000) PAX-8-PPARgammal fusion oncogene in human thyroid carcinoma. Science 289: $1357-1360$

Ledent C, Dumont J, Vassart G, Parmentier M (1991) Thyroid adenocarcinomas secondary to tissue-specific expression of simian virus-40 large Tantigen in transgenic mice. Endocrinology 129: 1391-1401

Macpherson I, Montagnier I (1964) Agar suspension culture for the selective assay of cells transformed by polyoma virus. Virology 23: $291-294$

Matias-Guiu X, Cuatrecasas M, Musulen E, Prat J (1994) p53 expression in anaplastic carcinomas arising from thyroid papillary carcinomas. J Clin Pathol 47: 337-339

Okamoto Y, Ozaki T, Miyazaki K, Aoyama M, Miyazaki M, Nakagawara A (2003) UbcH10 is the cancer-related E2 ubiquitin-conjugating enzyme. Cancer Res 63: 4167-4173
Pang XP, Hershman JM, Chung M, Pekary AE (1989) Characterization of tumor necrosis factor-alpha receptors in human and rat thyroid cells and regulation of the receptors by thyrotropin. Endocrinology 125: $1783-1788$

Pierotti MA, Bongarzone I, Borrello MG, Mariani C, Miranda C, Sozzi G, Greco A (1995) Rearrangements of TRK proto-oncogene in papillary thyroid carcinomas. J Endocrinol Invest 18: 130 - 133

Powell Jr DJ, Russell J, Nibu K, Li G, Rhee E, Liao M, Goldstein M, Keane WM, Santoro M, Fusco A, Rothstein JL (1998) The RET/PTC3 oncogene: metastatic solid-type papillary carcinomas in murine thyroids. Cancer Res 58: $5523-5528$

Russell JP, Powell DJ, Cunnane M, Greco A, Portella G, Santoro M, Fusco A, Rothstein JL (2000) The TRK-T1 fusion protein induces neoplastic transformation of thyroid epithelium. Oncogene 19: 5729-5735

Suarez HG, du Villard JA, Severino M, Caillou B, Schlumberger M, Tubiana M, Parmentier C, Monier R (1990) Presence of mutations in all three ras genes in human thyroid tumors. Oncogene 5: 565-570

Tallini G, Santoro M, Helie M, Carlomagno F, Salvatore G, Chiappetta G, Carcangiu ML, Fusco A (1998) RET/PTC oncogene activation defines a subset of papillary thyroid carcinomas lacking evidence of progression to poorly differentiated or undifferentiated tumor phenotypes. Clin Cancer Res 4: $287-294$

Tanaka J, Ogura T, Sato H, Datano M (1987) Establishment and biological characterization of an in vitro human cytomegalovirus latency model. Virology 161: $62-72$

Troncone G, Iaccarino A, Caleo A, Bifano D, Pettinato G, Palombini L (2003) p27 Kip1 protein expression in Hashimoto's thyroiditis. J Clin Pathol 56: 587-591

Wagner KW, Sapinoso LM, El-Rifai W, Frierson Jr HF, Butz N, Mestan J, Hofmann F, Deveraux QL, Hampton GM (2004) Overexpression, genomic amplification and therapeutic potential of inhibiting the UbcH10 ubiquitin conjugase in human carcinomas of diverse anatomic origin. Oncogene 23: 6621-6629

Wynford-Thomas D (1997) Origin and progression of thyroid epithelial tumours. Cellular and molecular mechanisms. Horm Res 47: $145-157$

Zeki K, Nakano Y, Inokuchi N, Watanabe K, Morimoto I, Yamashita U, Eto S (1993) Autocrine stimulation of interleukin-1 in the growth of human thyroid carcinoma cell line NIM 1. J Clin Endocrinol Metab 76: $127-133$ 